\title{
Lepton Flavour Mixing Matrix and CP Violation from Neutrino Oscillation Experiments
}

\author{
M. Fukugita ${ }^{1}$ and M. Tanimoto ${ }^{2}$ \\ ${ }^{1}$ Institute for Cosmic Ray Research, University of Tokyo, Kashiwa 2778582, Japan \\ ${ }^{2}$ Department of Physics, Niigata University, Niigata 950-2181, Japan
}

\begin{abstract}
The measurement of the charged-current ${ }^{8} \mathrm{~B}$ solar neutrino reaction on deuterium at the Sudbury Neutrino Observatory confirms the neutrino oscillation hypothesis for the solar neutrino problem, and the result favours the solution with large neutrino mixing angles. We demonstrate that the current neutrino oscillation data (including atmospheric and reactor neutrinos) are sufficient to construct the lepton flavour mixing matrix with a reasonable accuracy. We also infer the maximum size of $\mathrm{CP}$ violation effects consistent with the current neutrino oscillation experiments.
\end{abstract}


The Sudbury Neutrino Observatory (SNO) experiment [1] measured the charged current rate for ${ }^{8} \mathrm{~B}$ solar neutrino reactions on deuterium. The derived electron neutrino flux is lower by $3.3 \sigma$ than the neutrino flux obtained from the electron scattering event rate measured by the same experiment, and more accurately by Super-Kamiokande (SK) [2]. This difference is ascribed to the neutral-current induced reaction of three species of the left-handed neutrinos on electron scattering. The sum of the electron neutrino flux and that of other neutrinos, inferred from the difference, agrees with the electron neutrino generation rate expected in the sun [3], hereby confirming the neutrino oscillation hypothesis as the origin of the long-standing solar neutrino problem.

The SNO result also narrows the range of the neutrino oscillation parameters. The rate of the conversion of electron neutrinos to other neutrinos is somewhat larger than is expected in the optimal solutions within the neutrino oscillation hypothesis (e.g., [2],[4]) inferred from solar neutrino experiments available prior to the SNO experiment [5],[2], [6]. This indicates that the new optimal solution including the SNO data is located slightly inwards within the MSW [7] triangle. This makes the small angle solution (SMA) of the MSW effect disfavoured, since the absence of the distortion of the neutrino energy spectrum and of day-night effect observed at SK leaves only the small angle edge of the SMA to be allowed [2], contrary to the charged-current event at SNO indicates. For a similar reason the neutrino oscillation hypothesis in vacuo is now disfavoured. We are left with the large mixing angle solution (LMA) and the LOW solution [4] of the MSW conversion as the most likely solution to the solar neutrino problem. Detailed statistical analyses including the SNO data have already been made [8],[9],[10], and confirmed the picture we have sketched here.

In this Letter we present an analysis of the neutrino oscillation from a somewhat different view. We ask the question whether we can determine the lepton flavour mixing matrix using the presently available oscillation data, and answer this question positively. 
We also ask the magnitude of the $\mathrm{CP}$ violation effect allowed by the current neutrino oscillation experiment.

We write the neutrino mixing matrix

$$
\left|\psi_{\alpha}\right\rangle=U_{\alpha i}\left|\psi_{i}\right\rangle
$$

where $\alpha=e, \mu, \tau$ and $i=1,2,3$. The solar neutrino experiments tell us about $\left|U_{e 1}\right|$ and $\left|U_{e 2}\right|$, and the atmospheric [11],[12] and the K2K long base-line accelerator experiment [13] tell us about $\left|U_{\mu 3}\right|$. Another constraint is imposed on the element $\left|U_{e 3}\right|$ from the Chooz reactor experiment [14]. If errors are small this information should be sufficient to construct the full $3 \times 3$ lepton flavour mixing matrix (up to the phase) under the unitarity constraint on the matrix.

We consider all constraints at a $90 \%$ confidence level. We fix for simplicity the mass square difference between $\nu_{\tau}$ and $\nu_{\mu}$ to be $3.2 \times 10^{-3}(\mathrm{eV})^{2}$ as deduced from the atmospheric neutrino experiment of SK [11]. We determine allowed regions of the Kobayashi-Maskawa angles from the oscillation parameters and mapped them into physical mixing matrix elements. The present experimental information is not sufficient to constrain the phase factor. So we vary the phase $\phi$ between 0 and $\pi$ while searching for the allowed angles, adopting the matrix representation in Review of Particle Physics [15].

We take LMA for the solar neutrino mixing solution. The mixing matrix we derived reads

$$
U=\left[\begin{array}{ccc}
0.74-0.90 & 0.45-0.65 & <0.16 \\
0.22-0.61 & 0.46-0.77 & 0.57-1 / \sqrt{2} \\
0.14-0.55 & 0.36-0.68 & 1 / \sqrt{2}-0.82
\end{array}\right]
$$

where we are confined to the case of $\nu_{\mu}<\nu_{\tau}$, and only the modulus of the elements are shown. If we allow for $\nu_{\mu}>\nu_{\tau}$ the $(2,3)$ element takes $1 / \sqrt{2}-0.82$ and the $(3,3)$ element is $0.57-1 / \sqrt{2}$, i.e., the two elements are interchanged as the phase is unconstrained in our analysis. It is interesting to note that all matrix elements are reasonably constrained 
with the present neutrino oscillation data. This predicts the oscillation properties between any kinds of neutrinos. It is also interesting to notice that all elements except for $U_{e 3}$ are sizable. A marginal disparity is seen between the $U_{e 3}$ and $U_{\tau 1}$ elements, but more accurate input of $U_{e 2}$ is needed for a definitive conclusion. The small $U_{e 3}$ is the characteristic that has been predicted in some phenomenological neutrino mass matrix models [16],[17] prior to the Chooz experiment'.

Once this matrix is determined one can infer the maximum size of $\mathrm{CP}$ violation in the lepton sector. In the last few years the feasibility of detecting $\mathrm{CP}$ violation has been studied by a number of authors in view of long-baseline neutrino experiments with strong neutrino beams [18],[19]. In these studies only a few representative values of neutrino oscillation parameters, as written in terms of the angle representation, are adopted to examine experimental feasibility, and systematic parameter searches are not made. A more general analysis is straightforward with our neutrino matrix. The factor that represents the net CP violation effect can be written as [20]:

$$
J=\frac{\left|U_{e 1}\right|\left|U_{e 2}\right|\left|U_{\mu 3}\right|\left|U_{\tau 3}\right|\left|U_{e 3}\right|}{1-\left|U_{e 3}\right|^{2}} \sin \phi .
$$

This is evaluated using the lepton mixing matrix, but more conveniently expressed only with experimentally relevant quantities, as

$$
J=\frac{1}{4} \frac{\sqrt{\sin ^{2} 2 \theta_{\text {sol }}} \sqrt{\sin ^{2} 2 \theta_{a t m}}\left|U_{e 3}\right|}{1-\left|U_{e 3}\right|^{2}} \sin \phi,
$$

where $\theta_{\text {sol }}$ is the mixing angle that directly comes into solar neutrino oscillation, $\theta_{\text {atm }}$ is that for the atmospheric neutrino oscillation, and $\left|U_{e 3}\right|$ is directly constrained by the $\bar{\nu}_{e} \rightarrow \bar{\nu}_{\tau}$ oscillation experiment. Now it is easy to show that the maximum value of $J$ is given by

\footnotetext{
${ }^{1}$ Note that $(1,3)$ and $(3,1)$ elements are reversely expressed in [16] by convention.
} 


$$
J \leq 0.040 \sin \phi .
$$

Apart from the phase factor $\sin \phi$ the crucial factor that controls the feasibility of the $\mathrm{CP}$ violation experiment is $\left|U_{e 3}\right|$. This demonstrates the importance of the measurement of $\bar{\nu}_{e} \rightarrow \bar{\nu}_{\tau}$ oscillation beyond the current limit set by the Chooz experiment.

We remark that if the modulus of the matrix elements (other than the four we used as input) is experimentally determined, we can predict the $\mathrm{CP}$ violation phase $\phi$ as,

$$
\cos \phi=\frac{1}{2\left|U_{e 1}\right|\left|U_{e 2}\right|\left|U_{e 3}\right|\left|U_{\mu 3}\right|\left|U_{\tau 3}\right|}\left[\left(1-\left|U_{e 3}\right|^{2}\right)^{2}\left|U_{\mu 1}\right|^{2}-\left|U_{e 2}\right|^{2}\left|U_{\tau 3}\right|^{2}-\left|U_{e 1}\right|^{2}\left|U_{e 3}\right|^{2}\left|U_{\mu 3}\right|^{2}\right] .
$$

We do not repeat the discussion about actual $\mathrm{CP}$ violation effects given in the literature [19], but let us quote that the disparity of neutrino oscillation due to $\mathrm{CP}$ violation is given by [21]

$$
\begin{aligned}
\Delta P & =P\left(\bar{\nu}_{\mu} \rightarrow \bar{\nu}_{e}\right)-P\left(\nu_{\mu} \rightarrow \nu_{e}\right) \\
& =4 J f \leq 0.16 f \sin \phi,
\end{aligned}
$$

where $f=4 \sin \left(\Delta_{12} / 2\right) \sin \left(\Delta_{32} / 2\right) \sin \left(\Delta_{31} / 2\right)$ with $\Delta_{i j}=\Delta m_{i j}^{2} L / 2 E_{\nu}$ ( $L$ is the length of the baseline, and $E_{\nu}$ is the neutrino beam energy). The upper limit of $J$ corresponds to about 2/3 the value assumed in the analysis of Arafune et al.[19], but if it takes a value close to this limit the $\mathrm{CP}$ violation effect is probably visible.

So far we have discussed the case of the LMA solution. The experiments also allow the LOW solution, which are located close to the bottom of the MSW triangle, albeit the parameter range allowed by a $90 \%$ confidence is narrow, If LOW is the solution, the mixing matrix is

$$
U=\left[\begin{array}{ccc}
0.71-0.79 & 0.61-0.71 & <0.16 \\
0.34-0.65 & 0.42-0.70 & 0.57-1 / \sqrt{2} \\
0.25-0.58 & 0.32-0.63 & 1 / \sqrt{2}-0.82
\end{array}\right]
$$


This matrix is quite similar to the one given for LMA, but the constraint is slightly tighter because of a smaller allowed region for $U_{e 2}$. We obtain a $\mathrm{CP}$ violation $J$ factor similar to (5). The observable CP violation effect, however, contains the mass square difference, and the small $\Delta m_{12}^{2}$ of the LOW solution pushes the effect outside the range feasible with accelerator experiments. We should await the KamLAND experiment [22] for a decisive answer as to the selection between the two solutions.

\section{Acknowledgements}

We thank Tsutomu Yanagida for many valuable discussions over many aspects of neutrino physics, including the present work. 


\section{References}

??1] SNO Collaboration: Q. R. Ahmad et al., nucl-ex/0106015, Phys. Rev. Lett. in press (2001)

??2] Super-Kamiokande Collaboration: S. Fukuda et al. Phys. Rev. Lett. 86, 5651; $5656(2001)$

??3] J. N. Bahcall, M. H. Pinsonneault and S. Basu, astro-ph/0010346r2 (2001)

??4] J. N. Bahcall, P. I. Krastev and A. Yu. Smirnov, Phys. Rev. D58, 096016 (1998);

G. L. Fogli, E. Lisi and D. Montanino, Astropart. Phys. 9, 119 (1998)

??5] B. T. Cleveland et al., Astrophys. J. 496, 505 (1998)

??6] GALLEX Collaboration: W. Hampel et al. Phys. Lett. B447, 127 (1999); SAGE

Collaboration: J. N. Abdurashitov et al. Phys. Rev. C60, 055801 (1999)

??7] S. P. Mikheyev and A. Yu. Smirnov, Sov. J. Nucl. Phys. 42, 913 (1985); L. Wolfenstein, Phys. Rev. D17, 2369 (1978)

??8] G. L. Fogli, E. Lisi, D. Montanino and A. Palazzo, hep-ph/0106247

??9] J. N. Bahcall, M. C. Gonzalez-Garcia and C. Peña-Garay, hep-ph/0106258

??10] A. Bandyopadhyay et al. hep-ph/0106264

??11] Super-Kamiokande Collaboration: Y. Fukuda et al. Phys. Rev. Lett. 81, 1562 (1998); T. Toshito, in Proceedings of the 30th International Conference on High Energy Physics, Osaka, 2000, ed. C. S. Lim and T. Yamanaka (World Scientific, Singapore, 2001), Vol. 2, p. 913

??12] E. Peterson, in Proceedings of the 30th International Conference on High Energy Physics, Osaka, 2000, ed. C. S. Lim and T. Yamanaka (World Scientific, Singapore, 2001), Vol. 2, p. 907; F. Ronga et al. ibid p.910

??13] K2K Collaboration: S. H. Ahn et al. Phys. Lett. B511, 178 (2001)

??14] M. Apollonio et al., Phys. Lett. B466, 415 (1999)

??15] Particle Data Group, D. E. Groom et al., Eur. Phys. J. C15, 1 (2000) 
??16] M. Fukugita, M. Tanimoto and T. Yanagida, Prog. Theor. Phys. 89, 263 (1993)

??17] M. Fukugita, M. Tanimoto and T. Yanagida, Phys. Rev. D57, 4429 (1998)

??18] M. Tanimoto, Phys. Rev. D55, 322 (1997)

??19] J. Arafune, M. Koike and J. Sato, Phys. Rev. D 56, 3093 (1997); see also J. Arafune and J. Sato, Phys. Rev. D 55, 1653 (1997)

??20] C. Jarlskog, Phys. Rev. Lett. 551039 (1985)

??21] V. Barger, K. Whisnant, S. Pakvasa and R. J. N. Phillips, Phys. Rev. D 22, $2718(1986)$

??22] A. Suzuki, Nucl. Phys. B (Proc. Suppl.) 77, 171 (1999) 